\title{
A. Einleitung
}

\section{Ausgangspunkt: Die liedgeschichtliche Situation im letzten Drittel des 14. Jahrhunderts und die Forschungslage zu den Liedern des Korpus ,Mönch von Salzburg'}

Von der reichen und vielgestaltigen deutschsprachigen Liedkunst des 15. und 16. Jahrhunderts ist heute - die Kirchenlieder Luthers ausgenommen - so gut wie nichts mehr bekannt. Fragte man Zeitgenossen nach dem späten Mittelalter und der frühen Neuzeit, so würde kaum jemandem - auch wenn sie oder er für sich Anspruch nehmen könnte, literaturgeschichtlich weit überdurchschnittlich bewandert zu sein - ein Lied oder ein Gedicht in den Sinn kommen. Wenn überhaupt sind andere literarische Gattungen aus diesem Zeitraum präsent. ${ }^{1}$ Dagegen ist zumindest der Begriff des Minnesangs ziemlich fest mit dem des hohen Mittelalters verbunden und als Lyriker des 17. Jahrhunderts sind den literarisch Gebildeten die Namen Opitz, Hoffmannswaldau, Gryphius und Gerhardt vertraut. Betrachtet man aber, in welchem Umfang Lieder im 15. und 16. Jahrhundert handschriftlich aufgezeichnet und gedruckt wurden, kann man durchaus von Liederjahrhunderten sprechen. ${ }^{2}$ Für die zweite Hälfte des 14. Jahrhunderts macht die schlechte Überlieferungslage das Urteil schwieriger, die umfangreiche Liedproduktion und -aufzeichnung der späteren Zeiten müssen aber ihre Vorläufer im 14. Jahrhundert gehabt haben.

Die Gründe dafür, dass von der vielfältigen Liedkunst des Spätmittelalters und der frühen Neuzeit fast nichts mehr präsent ist, lassen sich in die Anfänge der Germanistik zurückverfolgen. Lieder aus diesen Jahrhunderten erweckten im frühen 19. Jahrhundert Interesse als Ausdrucksmittel eines im Kern als immer schon existent und unveränderlich gedachten, Volkes', man interessierte sich für sie als übergeschichtlich-kommune, Volkslieder‘. Nur als, Volkslied' wurde spätmittelalterliche und frühneuzeitliche Lyrik im 19. Jahrhundert Teil des literaturgeschichtlichen Kanons, nur als solches wurde sie einem breiteren Publikum bekannt. Nachdem zuerst die Vorstellung einer überzeitlichen Einheit, Volk', dann die des Kanons im emphatischen Sinn verabschiedet werden mussten, war diese frühe Art der Ka-

1 Das zeigt sich in neueren literaturgeschichtlichen Überblickswerken. Vgl. zu Hansers Sozialgeschichte der deutschen Literatur HüBNER 2004, Absätze 4, 5 u. 59. In der ,Geschichte der deutschen Lyrik' im ReclamVerlag ist im Beitrag von Holznagel 2004 das 14. und 15. Jh. behandelt. Ob und ggf. wie es mit den Liedtypen und Überlieferungstypen nach dem Ende des Mittelalters weiter ging (was z. B. gedruckte mit hsl. Liederbüchern gemeinsam haben), erfährt man nicht, weil der folgende Beitrag von KEMPER 2004 unter den Aspekt des Konfessionalismus gestellt ist. Liest man diese Geschichte der deutschen Lyrik am Stück, könnte man den Eindruck bekommen, zwischen dem Ende des 15. Jhs. und dem Barock hätte es nur Kirchenlieder gegeben. Vgl. dagegen Brunner 2013a, S. 410-434, und Cramer 1990, S. 308-341. Ein Blick in popularisierende Anthologien (z. B. ReIch-RaNicki [Hg.] 2002) bestätigt den Eindruck, dass es außer Luthers Liedern zwischen entweder Walther von der Vogelweide oder Oswald von Wolkenstein und dem Barockgedicht nur sehr wenige Blüten gab, die sich zu lesen lohnte.

2 Vgl. HüBner 2005b, S. 1, und Рetzsch 1959, S. 414-415. 
tegorisierung und Kanonisierung weder dem Forschungsinteresse noch dem allgemeinen Interesse an spätmittelalterlich-frühneuzeitlicher Lyrik förderlich. ${ }^{3}$

Dass die deutschsprachige Lyrik des 15. und 16. Jahrhunderts auch in der jüngeren Forschung eher wenig Aufmerksamkeit fand, liegt neben forschungsgeschichtlichen Gründen am Gegenstand selbst, der mit zum Großteil anonym überlieferten Texten einen insgesamt unübersichtlichen Eindruck erweckt. Hinderlich ist auch eine auf den ersten Blick verwirrende Vielzahl von Begriffen - Liebeslied (statt Minnelied), Liederbuch-Lied, Gesellschaftslied, Volkslied, Hofweise, politisches Lied, Meisterlied, Meistersingerlied -, die einerseits die Vielfalt der sozialen Gebrauchszusammenhänge reflektiert, andererseits einer Begriffsbildung geschuldet ist, die teilweise Begriffe aus anderen Zusammenhängen auf die Lyrik des 14., 15. und 16. Jahrhunderts überträgt oder verallgemeinert. Für Lieder des 16. Jahrhunderts gilt zudem, dass ihre Erforschung auf ausdifferenzierte Teildisziplinen - Mediävistik und Neuere Deutsche Literaturwissenschaft einerseits, Musikwissenschaft andererseits - verteilt ist. Dadurch geraten Zusammenhänge leicht aus dem Blick. Die Interessen und Fragestellungen der Nachbardisziplin - die man von der eigenen Warte aus jeweils für bestimmte Fragen zuständig hält - unterscheiden sich unter Umständen so sehr von den eigenen, dass Verständigung und Zusammenarbeit schwierig werden können.

Diese Arbeit will am Verständnis der Gattung Lied im späten Mittelalter und der frühen Neuzeit mitarbeiten. Sie bemüht sich, die literarische und die musikalische Seite des Liedes zu berücksichtigen; freilich zielt die Befragung der musikalisch-melodischen Seite auf primär literaturgeschichtliche Zusammenhänge. Im Zeitraum 1350-1600 setzt sie früh an, in den Jahrzehnten um 1400. Für diesen frühen Zeitabschnitt ist die literaturwissenschaftliche Forschungslage insgesamt besser als für das 16. Jahrhundert. Die germanistische Mediävistik hat sich ihm seit der zweiten Hälfte des 20. Jahrhunderts verstärkt zugewandt und viele Bereiche erschlossen, wobei hinsichtlich der Liedkunst die Schwerpunkte auf dem Traditionszusammenhang von Sangspruchdichtung, meisterlicher Liedkunst und Meistergesang einerseits, auf Oswald von Wolkenstein andererseits lagen. Diese beiden Schwerpunkte deuten zugleich ein Problem für die Erforschung des Zeitabschnitts an: In der zweiten Hälfte des 14. und im ersten Drittel des 15. Jahrhunderts gibt es wenig Liedaufzeichnungen, was die Beurteilung von Transformationsprozessen schwierig macht. Der Traditionszusammenhang von Sangspruchdichtung, meisterlicher Liedkunst und Meistergesang rückt hier in den Blick, weil bei ihm im Grundsatz Kontinuität besteht und sowohl vor 1350 als auch nach 1420 Sammelhandschriften vorhanden sind. Auf der anderen Seite sind einige Einzelœuvres bzw. -korpora tradiert - Mönch von Salzburg, Hugo von Montfort, Heinrich Laufenberg, Oswald von Wolkenstein -, die jedoch mehr oder weniger isoliert dastehen.

Betrachtet man aber die Überlieferungsgegebenheiten vor der Mitte des 14. Jahrhunderts und nach der Mitte des 15 . Jahrhunderts, wird deutlich, dass sich sowohl das Gat-

3 Zu diesem Absatz siehe HüBNER 2005b, S. 1-6. Zur Randständigkeit spätmittelalterlicher und frühneuzeitlicher Liedkunst vgl. auch die Einleitungen in Kiepe/Kiepe (Hgg.) 1972, S. 5-6, und Düwel (Hg.) 1978, S. 5-7. 
tungssystem der Lyrik als auch die soziokulturellen Kontexte von Liedkunst deutlich verändert haben. Dabei tritt

„immer wieder die Mitte des 14. Jahrhunderts als eine Wendemarke zwischen Spätmittelalter und Früher Neuzeit in den Blick. Dieser epochale Wandel läßt sich wohl in keinem anderen Gattungsbereich so gut ablesen wie in der Lyrik. “4

Auch aus musikhistorischer Sicht verdienen die zweite Hälfte des 14. und das erste Drittel des 15. Jahrhunderts Interesse, weil sich in dieser Zeit in Frankreich die Kunst der Ars nova etabliert, die nach und nach Auswirkungen für die musikalischen Entwicklungen in ganz Europa hat. Die Liedkunst im deutschsprachigen Raum partizipiert ab dem Ende des 14. Jahrhunderts allmählich an diesen musikalischen Entwicklungen, zeigt aber auch eigenständige Tendenzen. ${ }^{5}$

Als Ausgangspunkt und Zentrum dieser Arbeit wurde das Korpus der Lieder gewählt, die unter der Autorbezeichnung, Mönch von Salzburg' überliefert sind. Sie haben seit langem einen Platz in den literatur- und musikgeschichtlichen Handbüchern. ${ }^{6}$ Im Mönch-Korpus sind unterschiedliche Traditionslinien und Neuansätze deutschsprachiger Liedkunst präsent: Es finden sich geistliche und weltliche Lieder, unterschiedliche Formtraditionen einstimmiger Liedkunst, einfache/organale Mehrstimmigkeit und in einem Fall auch artifizielle Mehrstimmigkeit. ${ }^{7}$ Um genauer zu beschreiben, was sich bei jenem ,epochalen Wandel' abspielte, bietet das Mönch-Korpus zudem weitere Vorzüge:

Durch die Überlieferung in mehreren Sammelhandschriften des 15. Jahrhunderts handelt es sich um ein vergleichsweise geschlossenes Korpus. Die Zuschreibung an den Mönch und den Salzburger Hof ermöglichen eine historische und soziale Situierung.

Gegenüber dem Traditionsstrang von Sangspruch und meisterlichem Lied und dem ihm zuzurechnenden Liedœuvre Heinrichs von Mügeln (literarisch produktiv ca. 1350-1370), ${ }^{8}$ das in einer ebenfalls geschlossenen, darüber hinaus autornahen Überlieferung vorliegt, ist das Mönch-Korpus formal und inhaltlich vielgestaltiger. Dadurch ist es innerhalb des Kor-

4 JANOTA 2004, S. 145, zum Spätmittelalter allgemein ebd., S. 25-31; ähnlich BRUNNER 2013a, S. 302-303; Cramer 1990, S. 33-43, S. 215-219, S. 312-345, arbeitet stellenweise die Zusammenhänge zwischen älterer und jüngerer Liedkunst heraus, in der Gesamtanlage seiner Literaturgeschichte wird der Umbruch aber ebenfalls deutlich.

5 Vgl. SCHwindt 2004, S. 185-193, besonders S. 185-186 u. S. 192. Zur Rezeption französischer Liedkunst im deutschen Sprachraum und zu den vielgestaltigen Interessen, die zur Entstehung von Musikhandschriften führen konnten, siehe auch Kirnbauer 1996 und Kirnbauer 2001 (zusammenfassend S. 142-145, S. $197-198$ u. S. 211-220).

6 In chronologischer Folge: Riemann 1908, S. 84; Ludwig 1924, S. 171 (bei Riemann und Ludwig unter den ersten Ansätzen von Mehrstimmigkeit im deutschen Sprachraum); Ehrismann 1935, S. 595 (nur als Autor der geistlichen Lieder); UrSPRUnG 1931, S. 105; Rupprich 1970, S. 170-172; Michels 1977, S. 197; Möller/Stephan (Hgg.) 1991, S. 313 (als „späte Nachblüte des Minnesangs"); Cramer 1990, S. 36-38; Wörner 1993, S. 68, S. 78 u. S. 169; Holznagel 2004, S. 77-79; Janota 2004, S. 163-168; KNapp 2004, S. 448-478; BrunNer 2013a, S. 305-306 u. S. 314-315.

7 Vgl. Brunner/Ganser/Hartmann 1980/81, März 2005, Welker 1984/85.

8 Vgl. Stackmann 1981. 
pus möglich, die Schnittpunkte unterschiedlicher Liedtraditionen in den Blick zu bekommen.

Anders als die Euvres Hugos von Montfort $\left({ }^{*} 1357, \uparrow 1423\right)^{9}$ und Oswalds von Wolkenstein $\left(^{*} \text { ca. } 1376, \dagger 1445\right)^{10}$, die ganz oder annähernd wirkungslos blieben, zeigt schon eine oberflächliche Durchsicht der Überlieferung, dass die Lieder des Mönch-Korpus eine breite Wirkung entfalteten. Es gab in der zweiten Hälfte des 15. Jahrhundert offenbar ein Interesse an ihnen, das groß genug war, dass mehrere umfangreiche Sammelhandschriften angelegt wurden. Einzelne Lieder wurden vom Ende des 14. bis ins beginnende 16. Jahrhundert hinein handschriftlich aufgezeichnet, einige auch gedruckt. Die Streuüberlieferung ist bei den geistlichen Liedern außerordentlich breit. ${ }^{11}$ Betrachtet man das Mönch-Korpus vor dem Hintergrund der Lieder, die in den Liederbüchern des 15. Jahrhunderts stehen, ergibt sich oft der Eindruck, dass beim Mönch Liedtypen und Konzeptionen zum ersten Mal, erstmals in größerer Anzahl oder vergleichsweise früh auftreten. Allerdings kann der Eindruck des Frühen oder Erstmaligen auch Resultat der schlechten Liedüberlieferung in der zweiten Hälfte des 14. und im ersten Viertel des 15. Jahrhunderts sein.

Trotz der großen Wirkung der Lieder des Mönch-Korpus und ihrer Situierung in einer liedgeschichtlichen Sattelzeit ist die Forschung zum Mönch überschaubar. Auf die Edition der geistlichen Lieder durch Philipp Wackernagel im Rahmen seines „Deutschen Kirchenliedes $^{612}$ und der weltlichen durch Heinrich Mayer und Arnold Rietsch ${ }^{13}$ folgten Arbeiten zur Identität des Autors ${ }^{14}$ sowie literatur ${ }^{15}$ und musikhistorische ${ }^{16}$ Studien. Das Faksimile

9 Vgl. Wachinger 1983d und Hofmeister (Hg.) 2005.

10 Vgl. Sсhwob 1977, Wachinger 1989c, Spicker 2007, U. Müller 2010, U. Müller/Springeth (Hgg.) 2011 und die Beiträge des Tagungsbandes JOWG 19 (2012/13): Oswald von Wolkenstein im Kontext der Liedkunst seiner Zeit.

11 Übersichten und einzelne Funde zur Mönch-Überlieferung finden sich in (in chronologischer Folge): Spechtler 1963a, Spechtler 1963b, Röll 1970, Spechtler (Hg.) 1972, Spechtler 1977, d’Aronco 1980a, d’Aronco 1980b, Hayer/Spechtler 1980 (dazu März 1980/81), Zimmermann 1982a, ZimmerMANN 1982b, WACHINGer 1989a, MärZ (Hg.) 1999, Henkel 2008. Vgl. weiter die Zusammenstellungen im HSC unter ,Mönch von Salzburg: Lieder' <http://www.handschriftencensus.de/werke/2056> [10.02.2017], und ,Mönch von Salzburg: Guldein Abc'<http://www.handschriftencensus.de/werke/538> [10.02.2017]. Meine eigene Arbeitszusammenstellung von Textzeugen der geistlichen Lieder (Stand: Dezember 2011) umfasst 165 Einträge, ohne dass gezielt Kataloge und Handschriften auf weitere Textzeugen durchsucht wurden. Die Überlieferung reicht vom späten 14. bis ins frühe 16. Jh., von Sammelhandschriften bis zu Exzerpten aus einzelnen Liedern. Für Hinweise danke ich Gisela Kornrumpf, München, herzlich. Zu Exzerpten aus MönchLiedern siehe KornrumpF 2004b, Sp. 971-973, und Kornrumpf 2004e, Sp. 1477 u. Sp. 1479.

12 Wackernagel, KL, Bd. 2 (1867), S. 409-459, S. 461-463, S. 467-468 (Nr. 547-602, Nr. 605-606, Nr. 615). Bereits vor Wackernagel edierte Pfeiffer 1840 einige Lieder.

13 Mayer/Rietsch 1894/1896, vgl. März (Hg.) 1999, S. 1, Anm. 1 u. 2.

14 BAUERREISS 1934.

15 LoeWenstein 1932; NOACK 1941; RüDiger 1933; H. WAGNER 1898. Loewenstein mahnte bereits zu einer „kritischen Haltung gegenüber den ,haupthandschriften““ (LoEWENSTEIN 1932, S. 45), eine Einschätzung, die sich durch die Untersuchungen Wachingers bestätigen sollte, vgl. WACHINGER 1989a, S. 68.

16 SCHABasser 1936. 
der Mondsee-Wiener Liederhandschrift ${ }^{17}$ und die Edition der geistlichen Liedtexte durch Franz Viktor Spechtler stellten die Grundlage der neueren Forschung dar. ${ }^{18}$ Im Anschluss an Spechtlers Edition entstanden Studien zur Überlieferung ${ }^{19}$, Überblicksdarstellungen ${ }^{20}$, hymnologische Untersuchungen ${ }^{21}$ und Arbeiten zur Übersetzungstechnik ${ }^{22}$. Einzelne Übersetzungen des Mönch-Korpus fanden auch in jüngerer Zeit in Arbeiten zur Übersetzung und Verbreitung lateinischer Vorlagen Aufmerksamkeit. ${ }^{23}$ Literaturgeschichtliche Zusammenhänge wurden für einzelne Lieder genauer erforscht. G 45 wurde in einer Studie zu Cisioiani untersucht. ${ }^{24}$ Bei W 48 wurde die Vogelmetaphorik, ${ }^{25}$ bei W 5 das Verhältnis zur Gattung Tagelied analysiert. ${ }^{26}$ Beim Martinslied W 54* wurde unter Bezug auf Bachtins Theorie des Karnevalesken das Verhältnis von Heiligem und Weltlichem in der Bezugnahme auf Formulierungstraditionen herausgearbeitet ${ }^{27}$ und es wurden die geistlichen Kontrafakturen des Liedes untersucht. ${ }^{28}$ Bei W 55* wurde die Frage erörtert, ob das Lied Kontrafakt eines lateinischen Liedes sei. ${ }^{29}$ Innerhalb der Oswald-Forschung wurde der Mönch als ein Vorläufer Oswalds angeführt. ${ }^{30}$

In Auseinandersetzung mit und als Ergänzung zur Edition Spechtlers und zu den mit ihr verbundenen überlieferungsgeschichtlichen Arbeiten untersuchte Burghart Wachinger erneut die Überlieferungsverhältnisse für die einzelnen Lieder, trug eine beträchtliche Menge weiterer Streuüberlieferung zusammen und perspektivierte die Frage nach dem Autor und der Echtheit der Texte neu. ${ }^{31}$ Wachingers Resultate wurden durch die Untersuchung von Christoph März im Rahmen seiner Edition der Melodien und Texte der weltlichen Lieder ergänzt, sie bildet auch die Basis für die jüngere Auseinandersetzung mit den weltlichen Lie-

17 Heger (Hg.) 1968. Die Rezension durch Bertau 1975 erbrachte durch die Analyse der Wasserzeichen Fortschritte in der Datierung der Hs.

18 Spechtler (Hg.) 1972, zur weiteren älteren Forschung ebd., S. 3-8.

19 Bereits vor der Edition: Spechtler 1963a, Spechtler 1963b, Spechtler 1971, N. R. Wolf 1969; im Anschluss: Spechtler 1974, Spechtler 1980/81, Spechtler 1988, N. R. Wolf 1973, N. R. Wolf 1974 a.

20 Flotzinger 1995, Spechtler 1986, Spechtler 1995.

21 LipPHARdT 1961, LippHaRdt 1972c.

22 Bärnthaler 1983, Bärnthaler 1984, Behr 1985, Behr 1987, Reiffenstein 1984.

23 Krass 1998, S. 234-244, untersucht im Rahmen seiner Arbeit zu den Übersetzungen der Sequenz Stabat mater auch G 16 Maria stuend in swinden smerzen, Wennemuth 2003, S. 73-80, im Rahmen ihrer Studie zum Hymnus Christe, qui lux es et dies die Übertragung G 43 Christe, du bist liecht und der tag.

24 Hilgers 1979.

25 BenNewitz-BEHr 1983.

26 HirschBERg 1996.

27 Krass 1999.

28 Holznagel 2007.

29 Kesting 1973.

30 StÄBlein 1974. Den Zusammenhang zwischen Oswald und dem Mönch bestritt N. R. Wolf 1974b. Vgl. an jüngerer Literatur zu Oswald und dem Mönch: SpICker 2007, Register, s. v. ,Mönch von Salzburg'; BrunNER 2011; Hartmann 2011; Spechtler 2011; Wachinger 2010, vgl. zu Oswald, Kl. 78 und W 15 März (Hg.) 1999, S. 405-406.

31 WaCHINGER 1989a. 
dern des Mönchs. ${ }^{32}$ Auch wenn angesichts der breiten Überlieferung weitere Funde wahrscheinlich sind, dürften diese das bisher gewonnene Gesamtbild der Mönch-Überlieferung nicht umstürzend verändern. Auch die Untersuchung des Problems der Autorschaft kann im Rahmen des Möglichen als abgeschlossen gelten. Zur Textedition der geistlichen Lieder trat zuletzt als willkommene wie notwendige Ergänzung die Melodieedition durch Hans Waechter und Franz Viktor Spechtler hinzu. ${ }^{33} \mathrm{Zu}$ den Gegebenheiten der Überlieferung, der Person des Mönchs und der Situierung am Salzburger Hof sei im Folgenden das Wesentliche skizziert. $^{34}$

Acht Handschriften (A, B, C, D, E, F, G, J) bilden die Korpusüberlieferung. Sie überliefern eine größere Anzahl von Liedern des ,Mönchs'. Sechs von ihnen kennen den ,Mönch (munich, monachus) als Autor, ihm werden die Lieder entweder als Korpus zugeschrieben (A, E; C) oder bei einzelnen Liedern wird der ,Mönch“ als Autor genannt (D; F; G). Drei nennen als Auftraggeber den Salzburger Erzbischof Pilgrim II. von Puchheim und geben dem Mönch einen Namen, zwei nennen auch einen Orden (A: Herman, ein Münich Benedictiner Orden, die Lieder seien gemeinsam mit ainem laypriester herrn Martein ${ }^{35}$ entstanden; C: maister Johanns [später in der Handschrift: Hans] prediger ordens ${ }^{36}$; E: her Johanns ain Munich $^{37}$ ). Die acht Korpushandschriften sind in der zweiten Hälfte des 15. Jahrhunderts entstanden. ${ }^{38}$ Vier der Korpushandschriften (A, B, E; D) - jene vier, die auch Melodien enthalten, C, G, J und F enthalten nur die Texte - stammen aus demselben Skriptorium, ${ }^{39}$ das bisher nicht genau lokalisiert werden konnte. Die Redaktions- und Schreibarbeit an den Mönch-Handschriften muss in etwa in den Jahren 1450-1470 erfolgt sein. Innerhalb dieser Handschriften aus dem „Mönch-Zentrum“" ${ }^{40}$ gehören - wie Sonderfehler und -lesarten zeigen - A, B und E in der Textüberlieferung enger zusammen. Zu dieser Gruppe gehört auch die nicht aus dem Mönch-Skriptorium stammende Handschrift F. Handschrift D aus dem Mönch-Skriptorium (,Mondsee-Wiener Liederhandschrift') erweist sich ihnen gegenüber

32 März (Hg.) 1999. Zuvor erschienen die Auswahledition geistlicher und weltlicher Lieder mit Melodie Spechtler/Korth/Heimrath (Hgg.) 1980 (im Anschluss daran zur Form von W 3 Petzsch 1984, vgl. dazu März (Hg.) 1999, S. 374-376), und die Textedition der weltlichen Lieder mit nhd. Nachdichtungen Aigner/Spechtler (Hgg.) 1995. Eine jüngere Auswahledition für die musikalische Praxis liegt vor mit Spechtler/SeYwald (Hgg.) 2004.

33 Waechter/Spechtler (Hgg.) 2004. Die Melodien zu einzelnen Lieder sind zudem auch ediert in den GGdM und in Brunner/Hartmann, SpS.

34 Das Folgende gibt zusammenfassend und von direkten Zitaten abgesehen ohne Einzelnachweise die Ergebnisse von Spechtler (Hg.) 1972, S. 3-25; Wachinger 1989a, S. 77-137, und März (Hg.) 1999, S. 1-8 u. S. 43-50, wieder.

35 Zit. nach März (Hg.) 1999, S. 56-57.

36 Zit. nach SpechtLer (Hg.) 1972, S. 46, Abkürzungen aufgelöst.

37 Zit. nach März (Hg.) 1999, S. 74.

38 Zur Datierung und zum weiterem Inhalt aller Hss. vgl. auch die Einträge in den Katalogen Menhardt 1961/1962, K. Schneider 1978, K. SChneider 1984, K. Schneider 1996 und in den Datenbanken HSC und MANUSCRIPTA.AT.

39 Der briefliche paläographische Nachweis durch Karin Schneider zit. in WACHINGER 1989a, S. 77-79.

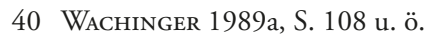


als eigenständiger. Eine weitere, enger zusammengehörige Gruppe bilden die Handschriften C, G und J, die etwas jünger sind als die Handschriften aus dem Mönch-Skriptorium. ${ }^{41}$

Drei weitere Handschriften überliefern ebenfalls eine größere Zahl von Liedern des Mönchs; sie nennen den Mönch als Autor, er wird hier aber in größere, nicht primär am Mönch interessierte Sammlungen eingereiht: in das ,Liederbuch der Clara Hätzlerin ' $(\mathrm{H})$, die Kolmarer Liederhandschrift $(\mathrm{K})$ und die Sterzinger Miszellaneen-Handschrift $\left(\mathrm{d} / \mathrm{St}^{42}\right)$. Die vermutlich um 1425 entstandene ${ }^{43}$ Sterzinger Miszellaneen-Handschrift belegt mit den Zuschreibungen Münch vō sāl diesen Autor zeitlich deutlich vor den Korpushandschriften. Die übrigen Handschriften zählen zur Streuüberlieferung. In ihnen sind teilweise drei oder vier, meist nur ein oder zwei Lieder des Mönch-Korpus enthalten, die Lieder sind überwiegend anonym überliefert.

Die Zuordnung des Mönchs zum Salzburger Hof Pilgrims II. ergibt sich nicht nur aus den Zuweisungen in den Handschriften, sondern auch aus einigen Liedern selbst. W 7 Der frëudensal: Dem allerlibsten schönsten weib ist als Brief Pilgrims an das libste[] E gestaltet und auf das Jahr 1392 datiert (W 7,3,9-12). W 19 Wier, wier der fünfczehent an der schar gibt sich als Brief des hofgesind [...] des hofs czu Salczburgk an die zurückgelassenen Frauen aus und trägt am Ende die Datierung 1387 (W 19,3,15). G 2 Plim gezartet ros an doren trägt das Akrostichon PYLGREIM ERCZBISCHOF LEGAT, G 3 Richer schatz der höchsten freuden das Akrostichon RICHERUS PLEBANUS IN RASTATT. Gemeint ist Reicher von Radstadt, der in Urkunden mehrfach als Pfarrer von Radstadt auftaucht $(1369,1378)$; als Kaplan am Salzburger Hof erscheint er 1380, als Hofmeister 1384 und 1385. Er ist wahrscheinlich vor 1410 verstorben. Da Pilgrim von 1366 bis 1396 als Erzbischof regierte, ergeben sich als Schaffenszeit des Mönchs die Jahrzehnte zwischen 1370 und 1400. Es ist nicht gelungen, den Mönch als eine namentlich bekannte Person am Salzburger Hof zu identifizieren, er war aber mit großer Wahrscheinlichkeit Teil der Hofgesellschaft und des engeren Zirkels um Pilgrim II.

Aus der Art der Überlieferung resultieren Schwierigkeiten, abzugrenzen, welche Texte dem Mönch zuzuschreiben sind. ${ }^{44}$ Grundsätzlich sind zwei Probleme zu unterscheiden. Die Korpushandschriften - insbesondere Handschrift A, die Korpushandschrift der geistlichen Lieder - weisen die Tendenz zur Erweiterung und Ergänzung auf. Die Zuweisung einzelner Lieder an den Mönch in Überschriften ist in den Korpushandschriften bisweilen anzutreffen, bisweilen nicht, eine Systematik wird nicht erkennbar, insgesamt sind Einzelzuweisungen in Überschriften eher selten. Die Zuschreibungen in Vorreden oder Beischriften sind auf das Lied-Korpus als Ganzes bezogen. Man muss daher damit rechnen, dass während des

41 Zur Datierung der Mönch-Hs. J (Wien, ÖNB. Cod. 3741) vgl. im Detail WACHINGer 1989a, S. 107-108.

42 Sigle d bei Wachinger 1989a, S. 2 u. S. 212, nach dem Verfahren Spechtlers (Hg.) 1972, S. 34; Sigle St bei März (Hg.) 1999, S. 123 u. S. 51.

43 Diese Datierung nach Siller 2001; zur Hs. vgl. unten, S. 136-137.

44 Außen vor bleiben in dieser Arbeit die Planetenkinderverse, die außerhalb der Korpushandschriften überliefert sind, aber in zwei Hss. dem Mönch zugeschrieben sind. Bei ihnen gibt es keine „Indizien für Sangbarkeit“ (Wachinger 1987a, Sp. 668). Vgl. weiter März (Hg.) 1999, S. 11, Anm. 15; Ausgabe auch in Aigner/ Spechtler (Hgg.) 1995, S. 243-244, zuletzt mit weiterer Lit. Sснмidt 2011. 
Prozesses des Sammelns, Ordnens und Redigierens aufgrund weiterer Interessen Texte in die Korpushandschriften aufgenommen und dem Mönch-Korpus einverleibt wurden, die nicht vom Mönch stammen bzw. nicht am Salzburger Hof entstanden sind. Andererseits ist damit zu rechnen, dass nicht in den Korpushandschriften enthaltene, anonym überlieferte Lieder dem Mönch zuzusprechen sind, wie das bei W 57* der Fall ist, das in d/St ohne Autornennung steht. ${ }^{45}$ Die zweite Frage ist, ob in Salzburg nur ein Dichter, der Mönch, tätig war. Handschrift A gibt in ihrer Vorrede zum Register an, dass der Mönch sampt ainem laypriester Martein die Lieder gemacht habe. ${ }^{46}$ Demnach hätte es am Salzburger Hof zwei an der Dichtung beteiligte Personen gegeben.

In der Frage nach einem oder mehreren Dichtern und der Frage nach später in die Korpushandschriften aufgenommenem Gut hat Spechtler in Zurückweisung älterer Versuche für die Übereinstimmung von Korpus und Euvre sowie die Zuschreibung des Gesamtœuvre an den Mönch votiert. ${ }^{47}$ Norbert Richard Wolf hielt Zweifel daran aufrecht. ${ }^{48}$ Burghart Wachinger sonderte für die geistlichen Lieder anhand von Kriterien der Überlieferung, der charakteristischen Liedtypen und des sprachkünstlerischen Anspruchs einen „sicheren Kernbestand“ 49 einerseits, eine Gruppe höchstwahrscheinlich erst später im Verlauf des Sammelns in die Korpushandschriften aufgenommener - unechter - Liedern andererseits aus. ${ }^{50}$ Zwischen beiden bleibt vor allem bei den Übersetzungen eine „Grauzone“ des Dichtens „à la Mönch", ${ }^{51}$ in der nicht zu entscheiden ist, ob man die Lieder dem einen Autor Mönch von Salzburg zu- oder absprechen kann. Ausgehend von der durch Wachinger beschriebenen stilistischen Bandbreite des überlieferten Korpus, ${ }^{52}$ hat Christoph März erwogen, dass diese stilistische Bandbreite nicht erst durch den Sammelprozess im 15. Jahrhundert zustande gekommen sein könnte, sondern dass die stilistischen Diskrepanzen bei einem guten Teil der Lieder bereits darauf zurückgehen, dass am Salzburger Hof mehrere Autoren tätig waren. ${ }^{53}$ Die Stilkritik ermöglicht es aber nicht, Lieder sicher dem einen oder anderen Autor zuzuschreiben; März hat daher in seiner Ausgabe auch nur stilistische Schichten unterschieden. ${ }^{54}$ Über die stilistischen Unterschiede eines am Salzburger Hof entstandenen Korpus hinaus muss man prinzipiell auch bei den weltlichen Liedern mit der Möglichkeit rechnen, dass

45 MärZ (Hg.) 1999, S. 505 (mit weiterer Lit.).

46 Zur möglichen Identifizierung vgl. SpechtLer (Hg.) 1972, S. 16-17.

47 Spechtler (Hg.) 1972, S. 11-14.

48 N. R. Wolf 1969, N. R. Wolf 1973, N. R. Wolf 1974a, N. R. Wolf 1974 b.

49 WACHINGER 1989a, S. 127, oder eine „relativ sichere Kerngruppe“ (ebd., S. 135). Zum Kernbestand zählen: G 1-G 7, G 9-G 13, G 16-G 18, G 20, G 21, G 23, G 27, G 28, G 33, G 36-G 43, G 45, G 47.

50 Wahrscheinlich unecht und erst im Sammelprozess dem Korpus integriert: G 8, G 22, G 24, G 34, G 35, G 46, G 44.

51 Siehe auch März 1991a, Zitate S. 115.

52 WACHINGER 1989a, S. 124-137.

53 März (Hg.) 1999, S. 3-8; vgl. auch März 1991a, S. 115, und WAchinger (Hg.) 2006, S. 951.

54 März (Hg.) 1999, S. 33-35. 
ein außerhalb Salzburgs entstandenes Lied einen Weg in die Korpushandschrift D finden konnte. ${ }^{55}$

Die weltlichen und die geistlichen Lieder sind deutlich differenziert. Sie verwenden unterschiedliche Strophenformen und überschneiden sich auch in den „Vorstellungsinhalten "56 nicht. Die vier Lieder, die zwischen den Sphären weltlich und geistlich stehen, verdanken diese Zwischenstellung ihrem Sujet. Der Text selbst muss hier keine Anstrengungen unternehmen, Weltliches und Geistliches zusammenzubringen: Die Martinslieder W 54* Von sand Marteins freuden: Wolauf, lieben gesellen vnuerczait und W 55* Ain radel: Martein, lieber herre sind Lieder an einen Heiligen, aber auch Fress- und Sauflieder, weil sein Festtag Gelegenheit zum Saufen und Fressen bot. ${ }^{57}$ Der Cisiojanus G 45 Besniten wirdigkleichen hat als Kalendermerkgedicht ebenso weltliche wie geistliche Zwecke. Der Tischsegen G 42 Allmächtig got, herr Jesu Christ hat ein geistlich-frommes Thema; aber weil er sich auf lebenspraktische Vollzüge bezieht oder für solche konzipiert war (Tischgebet), reicht er in profane Bereiche hinein. Das zeigt sich auch in der Aufzeichnung: In Handschrift D ist G 42 wie ein Teil der weltlichen Lieder als tenor bezeichnet ${ }^{58}$ und in Mensuralnotation aufgezeichnet, die sonst nur für die weltlichen Lieder verwendet wird ${ }^{59}$ zudem ist er einige Male im Verbund mit weltlichen Liedern des Mönch-Korpus überliefert. ${ }^{60}$

Vor dem Hintergrund, dass die Sichtung und Interpretation der Überlieferungsgegebenheiten sowie editorische Erschließung des Mönch-Korpus als großteils abgeschlossen, die Fragen nach Autor bzw. Autoren als soweit möglich geklärt gelten können, sind in jüngerer Zeit - neben Überblicksdarstellungen ${ }^{61}$ - mehrere Arbeiten zu weltlichen Liedern des Mönch-Korpus zu verzeichnen. ${ }^{62}$ Die umfangreichste Arbeit aus den letzten Jahren stammt von Christian Schneider, ${ }^{63}$ der sich an Fritz Peter Knapp ${ }^{64}$ anknüpfend mit der Funktion von Literatur im Kontext der höfischen Gesellschaft an einem klerikal geprägten Hof aus-

55 Anders als bei den geistlichen Liedern, von denen in allen acht Korpushss. eine größere Anzahl überliefert ist, sind die weltlichen Lieder fast nur in Hs. D gesammelt. Die anderen sieben Korpushss. enthalten wenige weltliche Lieder; zudem jene, die zwischen den Sphären geistlich und weltlich stehen. Ein spezifisches Interesse, das zur Erweiterung geführt hätte, lässt sich bei den weltlichen Liedern in Hs. D nicht so klar erkennen, wie bei den geistlichen Liedern in Hs. A. Von daher gibt es für den Verdacht späterer Integration ins MönchKorpus bei den weltlichen Liedern weniger Anhaltspunkte als bei einigen geistlichen. Zuletzt haben ohne neue Argumente WAEChTER 2005 und U. MüLLER 2009 wieder für einen Autor und die Einheit von Korpus und Euvre votiert.

56 März (Hg.) 1999, S. 6; vgl. WACHINGER 2010, S. 386-389.

57 Vgl. dazu ausführlich Krass 1999.

58 Siehe WACHINGER 1989a, S. 56.

59 Siehe Waechter 2005, S. 188-189.

60 Mönch-Hss. L, Re und Sb.

61 Holznagel 2004, S. 77-79; Janota 2004, S. 161-168; Kornrumpf 2010c; Knapp 2004, S. 448-478; März 2004a; Mattern 2016, S. 303-306; SpeChtLer 2001b; WACHINGER 1987a; Welker 2005.

62 Classen 2009; Holznagel 2007; Huber 1996; Hübner 2011a (vgl. auch Hübner 2005a und Hübner 2011b); Krass 1999; Lieb 2008; März 2005; Schmidt 2010 (auch zu G 22).

63 C. Schneider 2008 (vgl. dazu die Rezensionen von K. Wolf 2011 und Schnell 2010) und Schneider 2009.

64 Knapp 2004, S. 468-469. 
einandersetzte und der Frage nach dem Zustandekommen und der Bedeutung von hövescheit nachging. Im Vergleich mit dem Wiener Hof Albrechts I. beschrieb er den Salzburger Bischofshof als einen von den führenden Personengruppen her klerikal dominierten Hof, in dem die weltliche Liedkunst eine "Gesellschaftskunst ${ }^{\text {"65 }}$ war. Das Thema Liebe in den weltlichen Liedern habe einerseits zum Amüsement gedient, andererseits seien die Liedtexte aufgrund der sozialen und moralischen Konstellation - die Inhalte von Liebesliedern und erotischen Liedern konnten „in der Lebenswirklichkeit des geistlichen Hofes [...] nicht erlaubt ${ }^{\text {“66 }}$ sein - auch „Medium einer ebenso galanten wie allusionsreichen Kommunikation zwischen verschiedenen Personengruppen "67 gewesen. Schneider untersuchte überwiegend die weltlichen Lieder. Für seine Fragen nach Selbstdisziplinierung, sozialer Kontrolle, Körperbewusstsein und Geschlechterbeziehungen sind die geistlichen Lieder wenig aussagekräftig und wurden daher nur gestreift.

$\mathrm{Zu}$ den geistlichen Liedern im Besonderen sind in den letzten Jahrzehnten erneut Studien zu einzelnen Übersetzungen und zu Liedern auf eine Sequenzmelodie entstanden. ${ }^{68}$ Hans Waechters im Zusammenhang mit der Melodie-Edition entstandene Dissertation untersuchte für das ganze Korpus der geistlichen Lieder die Frage nach ihrem Gebrauch. ${ }^{69}$ Margarete Payer verfolgte in vier Liedern - darunter drei nicht-übersetzten - theologische und frömmigkeitsgeschichtliche Vorstellungen. ${ }^{70}$ Detailliert, fundiert und perspektivenreich situierte Britta Bußmann zwei Marienlieder des Mönch-Korpus im frömmigkeitsgeschichtlichen Kontext. ${ }^{71}$ Das Gesamtkorpus der Lieder des Mönchs unterzog zuletzt - im Hinblick auf das Liedœuvre Oswalds - Burghart Wachinger einer gattungstypologischen Betrachtung. Er zeigte, dass sich die Korpora als Ganzes besser verstehen und Einzelbeobachtungen genauer einordnen lassen, wenn man gattungstypologische Merkmale und die vielfältigen Gattungstraditionen um 1400 berücksichtigt; vor der „Folie“ des Mönchs lässt sich das Spezifische in Oswalds CEuvre genauer profilieren. ${ }^{72}$

Insgesamt dürfte auf der Hand liegen, dass eine Untersuchung der textlichen und melodischen Faktur sowie der Traditionszusammenhänge der geistlichen Lieder des MönchKorpus ein aussichtsreiches Unternehmen darstellt. Dabei liegt der Fokus auf den nichtübersetzten Texten. Dies ist nicht nur der spärlichen Forschungsliteratur zu diesen Liedern geschuldet. Vielmehr legen die nicht-übersetzten Lieder aufgrund ihrer unterschiedlichen Formen von sich aus die Frage nach vorausliegenden Text- und Musikgattungen und damit

65 C. SCHNeIDer 2008, S. 238.

66 Ebd., S. 244.

67 Ebd.

68 Fürbeth 2016, S. 431-438 (G 11); März 2004b (G 47); QuAST 2005 (G 5, G 47); Schiendorfer 1994 (G 29, G 30, G 31); A. SchneIder 2012, S. 120-121 (G 16).

69 Waechter 2005.

70 PAYER 2000: G 33 Kum, senfter trost heiliger geist, G 34 Kum, herr schepher heiliger geist (Veni creator spiritus), G 37 Got in drivaldikeit ainvalt, G 46 Eia herre got, was mag das gesein.

71 Bussmann 2016 (zu G 1); Bussmann 2017 (zu G 18); unergiebig sind die Beiträge Sснмidt 2016 und Mully 2011. Zu G 1 vgl. auch Degering 1916, S. 7-8 u. S. 11, und Krass 2014 (mit Übersetzung).

72 WACHINGER 2010, Zitat S. 386. 
die Frage nach den Traditionsfortschreibungen oder -umbrüchen nahe. Hierbei rücken die Lieder ins Zentrum, die weder übersetzt sind, noch die Melodie einer Sequenz verwenden. Die in der Forschung bereits artikulierte Vermutung, auch bei ihnen bestehe eine Nähe zu einer lateinisch-geistlichen Liedtradition, ${ }^{73}$ weckt zudem die Hoffnung, dass hier für einen vergleichenden Zugriff ein ausreichend großer Bestand an Gesängen mit Melodien überliefert sein und daher neben den formalen und inhaltlichen Aspekten auch an der Melodie angesetzt werden könnte.

73 Brunner 1983, S. 259-261. 